\title{
Principles for capturing the upstream effects of deep sills in low resolution ocean models
}

\author{
Lawrence J. Pratt, Misha Chechelnitsky \\ Woods Hole Oceanographic Institution, Woods Hole, MA 02543, USA \\ Received 30 October 1995; revised 14 May 1996; accepted 25 July 1996
}

\begin{abstract}
Principles for incorporating the upstream effects of deep sills into numerical ocean circulation models using nonlinear analytical hydraulic models are discussed within the context of reduced gravity flow. A method is developed allowing the upstream influence of a numerically unresolvable deep sill or width contraction to be reproduced. The method consists of placing an artificial boundary in the numerical model's overflowing layer at some distance upstream of the actual sill or width contraction of the deep strait. Given the model state at time $t$, the dependent flow variables are then predicted at the artificial boundary at time $t+\Delta t$ by using the method of characteristics in combination with quasi-steady hydraulic laws. The calculation requires the use of Riemann invariants and examples are given for a simple nonrotating flow and for rotating channel flow with uniform potential vorticity. The computation is considerably simplified by linearizing the relevant equations in the vicinity of the artificial boundary, resulting in a linear wave reflection problem. The reflection coefficients for the two cases are calculated and these can be used directly to numerically satisfy the boundary condition in a straightforward way.
\end{abstract}

\section{The problem}

Deep overflows are difficult to reproduce in global models of ocean circulation. Not only are the rapidly varying flows difficult to resolve, but the numerical model equations may be unable to faithfully reproduce the peculiar dynamics-hydraulic control, downstream mixing, etc.- characteristic of overflows. Hydraulic control may be an important

\footnotetext{
${ }^{1}$ Woods Hole Oceanographic Institution Contribution No. 9134
} 
regulator of the circulation and stratification of the deep flow upstream of the sill, while mixing has obvious consequences for the downstream flow. At least two simplified models of the downstream mixing have recently appeared (Jungclaus and Backhaus, 1994 and Price and Baringer, 1994) and these could, in principle, replace the local GCM treatment. In this paper we discuss how analytical hydraulic models may be used to reproduce the upstream effects of the sill without actually resolving the sill flow itself. Although this substitution is straightforward in purely steady models, it is not so in the presence of time dependence.

Referring to Fig. 1, which shows a deep overflowing layer of density $\rho_{2}$ underlying a layer of density $\rho_{1}$, we propose to remove the flow $0<x<x_{d}$ from the numerical model. We must therefore provide boundary conditions on the numerical model at $x=0$ and $x=x_{d}$. Our primary area of focus will be the boundary at $x=0$, which is fundamental to the hydraulic control of the upstream flow. The boundary condition at $x=x_{d}$, which specifies the degree of mixing taking place in the out flowing region in lee of the sill is not discussed and the reader is referred to recent 'outflow' models cited above.

Our aim is to discuss general principles and methodology rather than detailed procedures, as the latter will vary from one numerical model to the next. In some cases, implementation may even require further advances in rotating hydraulic theory. We therefore illustrate our methods using results from two idealized systems, both utilizing the $1 \frac{1}{2}$-layer approximation. The first involves simple nonrotating flow controlled by a sill and/or side contraction. Although not directly applicable to most deep passages, this model can be analyzed with minimal algebra and nicely demonstrates our main points. The second example is based on rotating flow with uniform potential vorticity which is hydraulically controlled in a deep passage with rectangular cross-section. Although idealized, this second model might be used to approximate the upstream effects of actual deep sills or width contractions where the overlying fluid could be considered locally inactive and the cross section locally rectangular, 'locally' meaning within a few grid points of the sill. For more general conditions, our guiding principles may still apply as detailed in Section 6.

Hydraulic control implies a relationship between the geometry of the control section (usually the most constricted section of the deep strait) and the variables characterizing

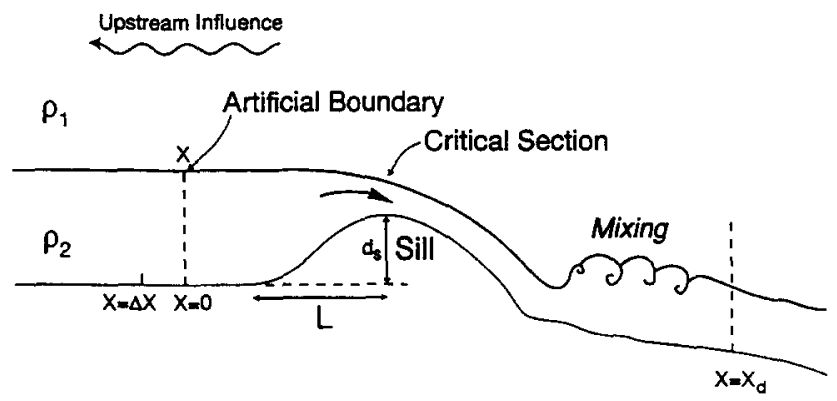

Fig. 1. Definition sketch. 
the upstream flow. For example, a single-layer, nonrotating flow in a channel with uniform width and a sill of height $d_{s}$ is constrained by the relation

$$
\frac{1}{2} u^{2}+g h-\frac{3}{2}(g u h)^{\frac{2}{3}}=g d_{s}
$$

where $h$ is the depth and $u$ the velocity of the upstream flow. (This relation is derived in Section 2.) In a noncontrolled flow $h$ and $u$ may be varied independently of each other; in a controlled flow they are linked by Eq. (1).

The property described above can be illustrated by the following experiment. Start with a steady, hydraulically controlled flow [with upstream values $h$ and $u$ therefore satisfying Eq. (1)]. At some location far upstream of the sill, generate a disturbance which attempts to establish new values $u$ and $h$ which do not satisfy Eq. (1). By doing so, one is attempting to establish a new flow inconsistent with the hydraulic control. As shown in Fig. 2(a), the disturbance will propagate and eventually reach the sill in the form of a wave front, with the initial flow downstream and the new flow upstream. When the wave reaches the sill a reflected wave is generated (Fig. 2(b)) which establishes yet another flow with depth $h_{2}$ and velocity $u_{2}$ and it can be shown that these new values satisfy Eq. (1). For the case of a noncontrolled flow the same experiment can be performed with the result (Fig. 2(c)) that the reflected wave is isolated and does nothing to alter the upstream state $u$ and $h$. (The schematic diagrams of Fig. 2 are based on actual integrations of the full shallow water equations by Pratt (1984)). In conclusion, hydraulic control is a process exercised through wave reflection, an idea central to its incorporation into GCMs.

Now suppose that the flow upstream of the obstacle has more complicated time-dependence, with many incident and reflected signals. We also assume that the wave lengths of all disturbances are large compared with the half-length $L$ of the sill (Fig. 1), so that the flow over the obstacle is quasisteady and Eq. (1) may be applied at any given

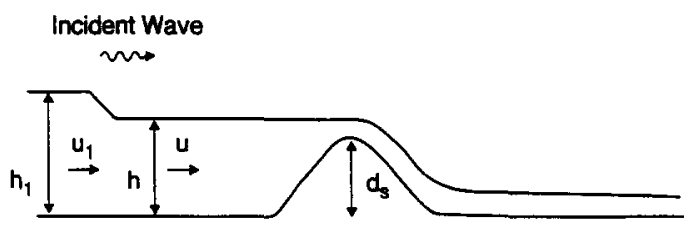

(a)

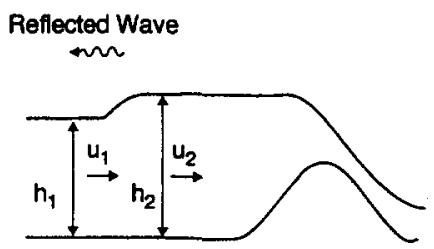

(b)

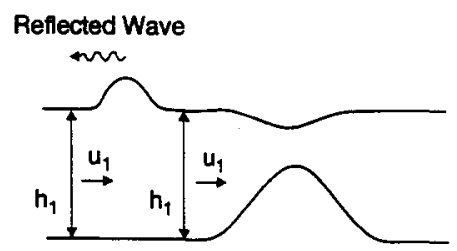

(c)

Fig. 2. Reflection of incident bore-like wave showing (a) the incident wave, (b) the reflected wave for the case of critically controlled flow and (c) the reflected wave for the case of no control. 
instant in time. Suppose further that the time-dependent and possibly nonlinear upstream state is calculated using a GCM with an artificial boundary at $x=0$ (Fig. 1). From a computational standpoint, the problem is this: Give $u$ and $h$ for all $x<0$ at some initial time $t_{0}$, what is the boundary condition (at $x=0$ ) at time $t_{0}+\Delta t$ ? Once this condition is known it should be possible to compute the remainder of the interior $(x<0)$ solution at $t_{0}+\Delta t$ using the applicable finite-difference or spectral scheme. Of course, certain time-stepping schemes may require the boundary conditions at $t_{0}+2 \Delta t$ or $t_{0}+\Delta t / 2$ say, but this does not greatly alter the procedure outlined below.

Since Eq. (1) provides only a relationship between $u$ and $h$, and not individual values, additional information is required to calculate the individual values $u\left(0, t_{0}+\Delta t\right)$ and $h\left(0, t_{0}+\Delta t\right)$. To proceed, one must rely on the property that hydraulically controlled flows can be influenced only by information originating upstream of the control section (here the sill). In terms of the flow at $x=0$, signals propagating in the downstream direction may be regarded as being independent while upstream propagating signals depend on Eq. (1) and provide no further information. The separation of upand downstream propagating information is particularly straightforward, even under nonlinear conditions, when the governing equations are locally hyperbolic. One may then use the method of characteristics and Riemann invariants to project incoming signals forward in time at $x=0$.

One can generally force the equations of motion to be hyperbolic near $x=0$ by making the approach from the upstream basin gradual and sufficiently narrow that all scales of $x$-variation, including wave lengths, are large compared with the channel width (see Fig. 3). In practice, imposing this geometry may lead to some local departures from actual bathymetry. Even when the flow has strong cross-channel variations, it is generally possible to separate this dependence from the $x$ - and $t$-dependence and define a hyperbolic system in terms of certain bulk characteristics. This procedure is well known in hydraulic theory and an example is given later in this paper.

In practice, the fluid overlying the lower layer will be partitioned into a number of layers or levels, the motion within computed numerically. If the bottom layer velocities developed at the sill are relatively large compared with the overlying fluid, it may be acceptable to decouple the lowest layer from the overlying fluid. One then imagines an artificial boundary to exist only within the lowest layer, so that the flow from $x=0$ to

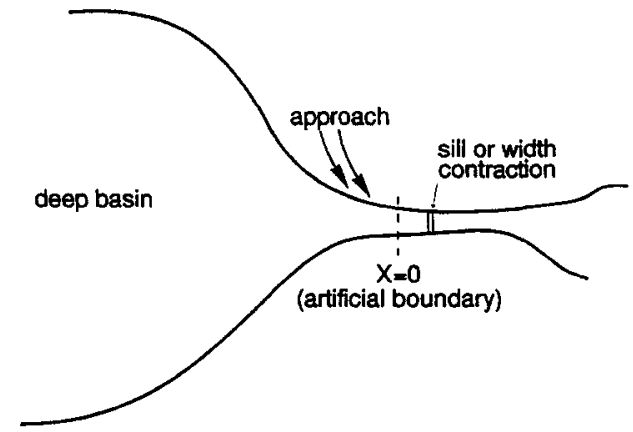

Fig. 3. Plan view of deep strait, the side wall boundaries corresponding to an isobath. 
the sill is removed from numerical treatment only in the lowest layer. Overlying motion is calculated using the normal numerical procedure or a modified form thereof.

Section 2 describes the procedure outlined above when the flow in the deep channel is nonrotating and uniform in cross-section. We then demonstrate the procedure using a problem for which a full analytical solution is known: linear wave reflection from the sill (Section 3). In Sections 4 and 5, this plan is repeated for the case of rotating channel flow with uniform potential vorticity in the cross-stream direction, a model we believe to be suitable for incorporation into GCMs. Aside from their intrinsic physical interest, the linear wave reflection calculations lead to an algebraically simple, approximate method for numerically satisfying boundary conditions.

\section{Nonrotating channel}

For purposes of illustration we first consider a nonrotating channel with rectangular cross-section and containing a flow with depth and velocity uniform in the cross-axis (y) direction. Upstream of the artificial boundary $(x<0)$ we assume that the channel width and bottom elevation have constant values $W$ and 0 . (The procedure for variable width and bottom elevation is included in the rotating model of Section 4.) Downstream of the artificial boundary $x>0$ the channel contains a sill of elevation $d_{s}$ and side contraction at which the width is $W_{s}$, here assumed to lie at the same value of $x$. The flow in this region is assumed quasisteady.

The equations governing the time-dependent flow in the approach are

$$
\begin{aligned}
& \frac{\partial u}{\partial t}+u \frac{\partial u}{\partial x}+g \frac{\partial h}{\partial x}=0 \\
& \frac{\partial h}{\partial t}+\frac{\partial(u h)}{\partial x}=0
\end{aligned}
$$

where $g$ denotes gravitational acceleration (1-layer model) or reduced gravitational acceleration ( $1 \frac{1}{2}$-layer model).

Using a procedure described by Whitham (1974) it is possible to write Eq. (2) and Eq. (3) as

$$
\frac{d_{ \pm} R_{ \pm}}{d t}=0
$$

where

$$
\frac{d_{ \pm}}{d t}=\frac{\partial}{\partial t}+\left[u \pm(g h)^{\frac{1}{2}}\right] \frac{\partial}{\partial x}
$$

and

$$
R_{ \pm}=u \pm 2(g h)^{\frac{1}{2}}
$$


$R_{ \pm}$are the Riemann invariants corresponding to the two gravity waves of the system (identified by ' + ' or ' - ') one of which propagates with speed $u+(g h)^{\frac{1}{2}}$ and the other with speed $u-(g h)^{\frac{1}{2}}$. In the absence of width or bottom elevation variations, $R_{+}$and $R$ are conserved following these signals. The local velocity and depth can be related to $R_{ \pm}^{-}$by

$$
\begin{aligned}
& u=\frac{1}{2}\left(R_{+}+R_{-}\right) \\
& g h=\frac{1}{16}\left(R_{+}-R_{-}\right)^{2} .
\end{aligned}
$$

In the quasisteady region immediately downstream of the artificial boundary, the energy (Bernoulli function) and volume transport are approximately uniform over $x$ at any $t$, and we may therefore write

$$
\frac{u_{b}^{2}}{2}+g h_{b}=\frac{u_{s}^{2}}{2}+g\left(h_{s}+d_{s}\right)
$$

and

$$
u_{b} h_{b} W=u_{s} h_{s} W_{s}
$$

where the subscript $b$ denotes values at $x=0$ and $s$ denotes values at the sill/width contraction. At the sill the flow is critical:

$$
u_{s}=\left(g h_{s}\right)^{\frac{1}{2}}
$$

Eliminating $u_{s}$ and $h_{s}$ between Eq. (6) and Eq. (8) yields the relation

$$
\frac{1}{2} u_{b}^{2}-\frac{3}{2}\left(g u_{b} h_{b} W / W_{s}\right)^{\frac{2}{3}}+g h_{b}=g d_{s}
$$

which is essentially Eq. (1) generalized to include width variations. Substitution of Eq. (5a) and Eq. (5b) into Eq. (9) yields

$$
\left[\left(R_{+}+R_{-}\right)^{2}+2\left(R_{+}-R_{-}\right)^{2}-8 g d_{s}\right]=\frac{1}{2}\left(\frac{3}{32}\right)^{2}\left[\left(R_{+}+R_{-}\right)\left(R_{+}-R_{-}\right)^{2} W / W_{s}\right]^{3}
$$

in which $R_{+}$and $R_{-}$are evaluated at $x=0$.

The computational procedure for determining the values of $u_{b}\left(t_{0}+\Delta t\right)$ and $h_{b}\left(t_{0}+\right.$ $\Delta t)$ from the known $u\left(x, t_{0}\right)$ and $h\left(x, t_{0}\right)$ over $x \leq 0$ is straightforward. First, calculate the characteristic speed $u\left(x, t_{0}\right)+\left[g h\left(x, t_{0}\right)\right]^{\frac{1}{2}}$ at several grid points to the immediate left of the artificial boundary $x=0$. From these estimate the origin $\left(x=x_{0}\right)$ of the characteristic curve $\mathrm{d} x / \mathrm{d} t=u(x, t)+[g h(z, t)]^{\frac{1}{2}}$ which passes through $x=0$ at time $t_{0}+\Delta t$. (Since the numerical scheme will generally prevent information from propagating more than one grid point in one time step, the interpolated value will lie between the last two grid points.) Next, compute the value of $R_{+}=u\left(x_{0}, t_{0}\right)+2\left[g h\left(x_{0}, t_{0}\right)\right]^{\frac{1}{2}}$ which corresponds to this curve. Since $R_{+}$is conserved, it follows that the value of 
$u\left(0, t_{0}+\Delta t\right)+2\left[g h\left(0, t_{0}+\Delta t\right)\right]^{\frac{1}{2}}$ has been established. Third, calculate the value of $R\left(0, t_{0}+\Delta t\right)$ from Eq. (10). The boundary values of both $R_{+}$and $R$ are now known at $t_{0}{ }^{-}+\Delta t$ and the corresponding $u$ and $h$ can be computed from Eq. (5a) and Eq. (5b). Once the interior flow field at $t_{0}+\Delta t$ has been numerically determined, a corrective procedure can be implemented if desired by re-estimating the path of the original characteristic curve.

\section{Wave reflection}

We now turn our attention to an example of the procedure just described, namely, linear wave reflection from a sill. There are several reasons for choosing this particular calculation, one being its use as an illustration of the numerical procedure in a familiar setting where the analytical solution can also be found. Second, the result of the calculation, the reflection coefficient $R_{c}$, can be used as a basis for a substitute to the procedure described in the previous section. If the approach flow can be approximated as the sum of a slowly varying background $(h=H, u=U)$ and linear perturbations $\left(\eta(x, t), u^{\prime}(x, t)\right)$ then the characteristic curves are straight lines and Eq. (10) is replaced by a simple linear relation between incident and reflected wave fields. Although the simplification in the procedure for forecasting the boundary conditions is modest, the simplification of the procedure described later for rotating channel flow is enormous. Finally the linear reflection problem is of intrinsic interest from the standpoint of time-dependent sill flow and, in fact, leads to some unexpected results.

Suppose now that the approach region of the channel has uniform width $W$ and extends a great distance upstream of the artificial control. Downstream the channel may narrow, reaching its minimum width $W_{s}$ at the sill section. At some initial instant the upstream flow is steady and uniform with depth and velocity $H$ and $U$ except for the presence of an isolated wave of small amplitude propagating towards the artificial boundary. The object is to calculate the reflected wave.

As a starting point, consider an incident or forward propagating disturbance moving into a steady region with velocity $U$ and depth $H$. If we denote the disturbance velocity and depth by $U+u_{I}$ and $H+\eta_{I}$ then

$$
\begin{aligned}
& R_{+}=U+u_{I}+2\left[g\left(H+\eta_{I}\right)\right]^{\frac{1}{2}}, \\
& R_{-}=U+u_{I}-2\left[g\left(H+\eta_{I}\right)\right]^{\frac{1}{2}}=U-2(g H)^{\frac{1}{2}} .
\end{aligned}
$$

From the second relation we have

$$
u_{l}=2\left[g\left(H+\eta_{I}\right)\right]^{\frac{1}{2}}-2(g H)^{\frac{1}{2}}
$$


and therefore

$$
R_{+}=2 u_{I}+U+2(g H)^{\frac{1}{2}}=4\left[g\left(H+\eta_{I}\right)\right]^{\frac{1}{2}}+U-2(g H)^{\frac{1}{2}}
$$

The same procedure can be carried out with a backward propagating wave with velocity $U+u_{R}$ and depth $H+\eta_{R}$, leading to

$$
R_{-}=2 u_{R}+U-2(g H)^{\frac{1}{2}}=-4\left[g\left(H+\eta_{R}\right)\right]^{\frac{1}{2}}+U+2(g H)^{\frac{1}{2}} .
$$

For backward and forward propagating disturbances moving through each other we may simply regard $u_{l}$ and $\eta_{l}$ as proxies for $R_{+}$with the interpretation that they represent the actual velocity and displacement when the backward propagating disturbance is absent. The same interpretation applies to $R_{-}, u_{R}$, and $\eta_{R}$. For $(\eta \ll H, u \ll U)$ Eq. (11a), Eq. (11b) reduce to

$$
\begin{aligned}
& u_{I}=g^{\frac{1}{2}} H^{-\frac{1}{2}} \eta_{I} \\
& u_{R}=-g^{\frac{1}{2}} H^{-\frac{1}{2}} \eta_{R} .
\end{aligned}
$$

In addition Eq. (5a), Eq. (5b) can be used to show that $u_{I}+u_{R}$ and $\eta_{I}+\eta_{R}$ are the total perturbation velocity and depth associated with two linear waves passing through each other.

In the numerical version of this analytical calculation Eq. (12a), Eq. (12b) would be hidden. The algorithm would simply identify the values of $R_{+}$contained in the incident wave, project them along characteristic curves to the artificial boundary, and calculate the $R$ values determining the reflected wave from Eq. (10). Alternatively, one could analytically calculate the reflection coefficient

$$
R_{c}=\eta_{R}(0, t) / \eta_{l}(0, t)
$$

by substituting Eq. (11a), Eq. (11b) into Eq. (10), linearizing, and using Eq. (12a), Eq. (12b). After some algebraic simplification the result

$$
R_{c}=\frac{\left(1+F_{d}\right)\left[1-F_{d}^{\frac{1}{3}}\left(W_{s} / W\right)^{\frac{2}{3}}\right]}{\left(1-F_{d}\right)\left[1+F_{d}^{\frac{1}{3}}\left(W_{s} / W\right)^{\frac{2}{3}}\right]}
$$

is obtained, where $F_{d}$ is the Froude number of the background approach flow:

$$
F_{d}=U /(g H)^{\frac{1}{2}} \text {. }
$$

An alternative numerical procedure, then, would be to identify $u_{I}$ and $\eta_{I}$ from the calculated upstream values of $R_{+}$and the relation Eq. (11a), project their values to the 
artificial boundary along straight characteristic curves $\mathrm{d} x / \mathrm{d} t=U+(g H)^{\frac{1}{2}}$, compute $\eta_{R}(0, t)$ using the reflection coefficient Eq. (13), and find $u_{R}(0, t)$ using Eq. (12b). The projected boundary values of depth and velocity would therefore be $H+\left(1+R_{c}\right) \eta_{I}$ and $U+(g / H)^{\frac{1}{2}}\left(1-R_{c}\right) \eta_{l}$. Such a procedure would be valid as long as the variations in $u$ and $h$ in the region immediately upstream of the artificial boundary are small compared with the mean or background values $H$ and $U$. Note that $U$ and $H$ can be redefined at the start of each time iteration to account for a background with slow temporal variations.

Fig. 4(b), Fig. 4(c) shows plots of the reflection coefficient $R_{c}$ for subcritical upstream flows $0<F_{d}<1$ and for various values of the width constriction $\left(W_{s} / W\right)$. (The analysis is invalid and would probably be of little geophysical relevance for

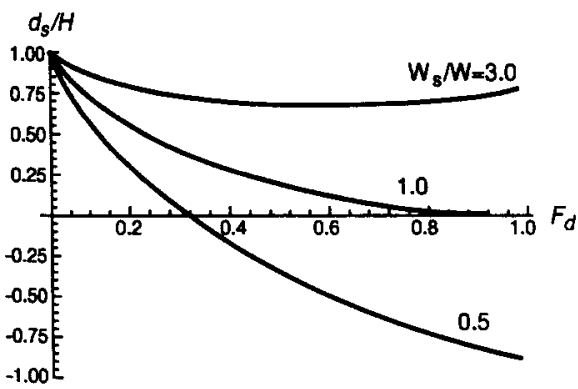

a
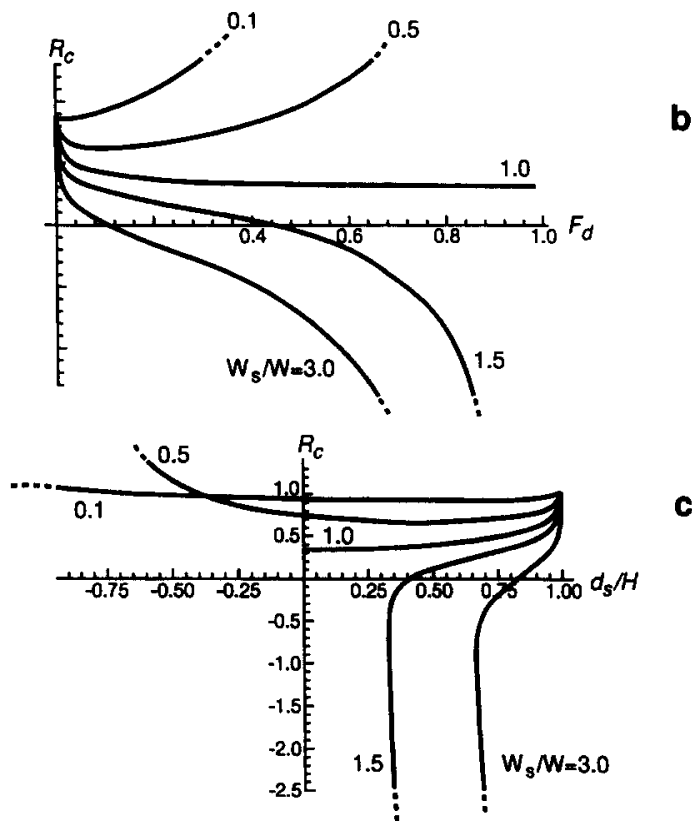

Fig. 4. (a) The relationship (14) plotted for three values of $W_{s} / W$. (b) The reflection coefficient $R_{c}$ vs. $F_{d}$ for various $W_{s} / W$. All but the $W_{s} / W=1.0$ curve grow without bound as $F_{d} \rightarrow 1$. (c) $R_{c}$ vs. $d_{s} / H$. Again, all but the curve $W_{s} / W=1.0$ go off to $+\infty$ or $-\infty$. 
$F_{d}>1$.) To interpret the results it is helpful to keep in mind the relation between $F_{d}$ and the relative obstacle height

$$
\frac{1}{2} F_{d}^{2}-\frac{3}{2} F_{d}^{\frac{2}{3}}\left(W_{s} / W\right)^{-\frac{2}{3}}+1=d_{s} / H
$$

which is nothing more than the dimensionless version of Eq. (9). This relationship is plotted in Fig. 4(a) for several values of $W_{s} / W$. For near zero values of $F_{d}$, where the obstacle occupies most of the depth range of the approach flow $\left(d_{s} / H \cong 1\right)$, waves are almost completely reflected $\left(R_{c} \rightarrow 1\right)$. For larger values of $F_{d}$ the behavior of $R_{c}$ is more subtle, particularly for cases of width contractions or expansions (values of $W_{s} / W \neq 1$ ), and we therefore describe the case $W_{s} / W=1$ first. As $F_{d}$ increases from zero, the portion of the total layer depth occupied by the obstacle decreases (middle curve in Fig. 4(a)) and it is not surprising that the value of $R_{c}$ decreases as well (middle curve in Fig. 4(b) and Fig. 4(c)). However, as the obstacle height vanishes, which occurs in the limit $F_{d} \rightarrow 1$, the reflection coefficient does not vanish, approaching the value $R_{c}=\frac{1}{3}$ instead. This unexpected result is a consequence of the fact that the upstream flow becomes critical as $F_{d} \rightarrow 1$, creating a near resonant state in which the reflected waves become stationary. Even the slightest variation in topography is able to produce a finite reflection. In fact, the only feature that keeps the reflected amplitude from blowing up is the fact that the sill height vanishes as $F_{d} \rightarrow 1$. Nonlinear theories for small but finite $d_{s}$, (e.g. Pratt, 1984; Grimshaw and Smyth, 1986) have shown that no steady solutions with the given uniform upstream conditions exist as $F_{d} \rightarrow 0$.

For cases of finite width contractions the behavior of $R_{c}$ is more complicated. If the sill section is constricted $\left(W_{s} / W<1\right)$ the value of $d_{s} / H$ decreases from unity (at $F_{d}=0$ ) to a negative value (at $F_{d}=1$ ), indicating that the sill elevation has become negative (lower curve in Fig. 4(a)). In the limit $F_{d}=1$, critical flow exists upstream of the control section of the channel, the latter consisting of a width contraction and a depression in the bottom. As shown by the $W / W_{s}=0.5$ curve in Fig. $4(\mathrm{~b}), R_{c}$ decreases from a value of unity at $F_{d}=0$ to a minimum of $R_{c} \cong 0.7$ at $F_{d} \cong 0.1$ and then increases without bound as $F_{d} \rightarrow 0$. The $W / W_{s}=0.5$ curve in Fig. 4(c) shows corresponding behavior as $d_{s} / H$ decreases. A range of upstream flows therefore exists for which the reflected wave has larger amplitude than the reflected wave, possible whenever $W_{s} / W \neq 1$.

Cases of width expansion are even more intriguing, as demonstrated by the $W_{s} / W=$ 3.0 curves of Fig. 4. The plot of $d_{s} / H$ versus $F_{d}$, in Fig. 4(a) has a minimum at $F_{d} \cong 0.5$, showing that a given upstream depth does not necessarily determine a unique upstream flow. Thus the plot of $R_{c}$ as a function of $d_{s} / H$ (e.g. the Fig. 4(c) curve with $W_{s} / W=3.0$ ) does not yield a single valued relation. As $d_{s} / H$ is decreased from unity, $R_{c}$ also decreases until the minimum $d_{s} / H$ is encountered. Proceeding further downwards along the curve causes $d_{s} / H$ to increase as $R_{c} \rightarrow-\infty$. The relationship between $R_{c}$ and $F_{d}$ continues to be single valued in this case as shown by the $W_{s} / W=3.0$ curve in Fig. 4(b). Note that situations in which no reflected wave is generated $\left(R_{c}=0\right)$ is possible whenever $\left(W_{s} / W\right)>1$. In such cases, the relationship between velocity and displacement in the incident wave exactly satisfies the hydraulic relation. 
Values of $\left|R_{c}\right|>1$ do not imply wave overreflection of energy, a finding which would imply flow instability. As we now show, the increase in reflected wave amplitude is accompanied by a sufficient decrease in wave length that the total reflected energy is less than the incident energy. Suppose that an isolated incident wave approaches the artificial boundary at $t=0$, resulting in an isolated reflected wave at $t=t_{0}$. Following Hayashi and Young (1987) or Ripa (1989) we define the incident disturbance energy as

$$
E_{I}=\frac{1}{2} \int_{-\infty}^{0}\left(H u_{I}^{2}+g \eta_{I}^{2}+2 U u_{I} \eta_{I}\right)_{t=0} d x .
$$

For a flow containing only an incident wave, $E_{I}$ is the difference between the total energy of the disturbed and undisturbed upstream flow (neglecting terms of order amplitude cubed). A similar definition may be made of the reflected disturbance energy $E_{R}$. Using Eq. (12a), Eq. (12b) the ratio of the two energies may be written as:

$$
R_{e}=\frac{E_{R}}{E_{I}}=\frac{\int_{-\infty}^{0}\left(1+F_{d}\right) \eta_{R}^{2}\left(x, t_{0}\right) d x}{\int_{-\infty}^{0}\left(1+F_{d}\right) \eta_{l}^{2}(x, 0) d x} .
$$

The two $x$ - integrals may be converted to time integrals at $x=0$ by projecting the values of $\eta_{I}$ and $\eta_{R}$ along characteristics. Hence

$$
R_{e}=\frac{\left(1-F_{d}\right)^{2} \int_{0}^{\infty} \eta_{R}^{2}(0, t) d t}{\left(1+F_{d}\right)^{2} \int_{0}^{\infty} \eta_{l}^{2}(0, t) d t}=\frac{\left(1-F_{d}\right)^{2}}{\left(1-F_{d}\right)^{2}} R_{c}^{2}=\frac{\left(1-F_{d}^{\frac{1}{3}}\left(W_{s} / W\right)^{\frac{2}{3}}\right)^{2}}{\left(1+F_{d}^{\frac{1}{3}}\left(W_{s} / W\right)^{\frac{2}{3}}\right)^{2}} .
$$

The value of $R_{e}$ is thus $\leq 1$. It is worth noting that if the cross term $\left(U \eta_{I} u_{I}\right.$ in Eq. (15)) is incorrectly left out of the definition of energy the resulting quantity, commonly referred to as wave energy, can increase during the reflection process. Note that the disturbance energy flux can be obtained by dividing Eq. (15) by the wave period. Since the reflected and incident periods are identical, $R_{e}$ can be interpreted as a disturbance energy flux coefficient.

It may sometimes be desirable to formulate a reflection coefficient in terms of mass or volume. Again consider an isolated incident wave at $t=0$ which partially reflects resulting in an isolated reflected wave at $t=t_{0}$. The net displacements associated with each disturbance are

$$
M_{I}=\int_{-\infty}^{0} \eta_{I}(x, 0) d x M_{R}=\int_{-\infty}^{0} \eta_{R}\left(x, t_{0}\right) d x
$$

and it can be shown through integration of the linearized form of Eq. (3) over $0 \leq t \leq t_{0}$ and $-\infty<x \leq 0$ that

$$
R_{V}=\frac{M_{R}}{M_{l}}=\frac{1-F_{d}}{1+F_{d}} R_{c}=\frac{1-F_{d}^{\frac{1}{3}}\left(W_{s} / W\right)^{\frac{2}{3}}}{1+F_{d}^{\frac{1}{3}}\left(W_{s} / W\right)^{\frac{2}{3}}} .
$$

Fig. 5 contains plots of $R_{v}$ as a function of $d_{s} / H$, where again Eq. (14) has been used to write $F_{d}$ in terms of $d_{s} / H$. As can be seen directly from Eq. (17), $\left|R_{v}\right| \leq 1$ for 


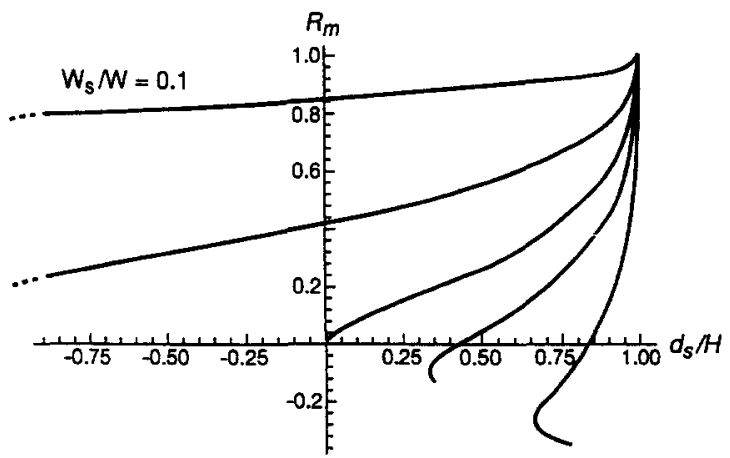

Fig. 5. The volume reflection coefficient $R_{l}$ as a function of $d_{s} / H$ using (14) and (17). The $W_{s} / W$ values for each curve are (from top to bottom) $0.1,0.5,1.0,1.5$ and 3.0. The upper two curves continue to the left of $d_{s} / H=-0.78$ where they have been discontinued. The curves $W / W_{s}=1.5$ and 3.0 terminate as shown.

all $0 \leq F_{d} \leq 1$ and therefore the reflected wave carries less absolute displacement than the incident wave. Also note that $M_{R}=0$ if $M_{I}=0$ so that no transport rectification is possible. (Rectification would require that we consider nonlinear effects in the upstream wave field, as done by Helfrich (1995), in connection with a two-layer flow.)

Finally, we take a few lines to formalize the 'quasisteady' approximation. The general idea is that over the obstacle, the hydraulic balance between terms like $u(\partial u / \partial x)$ and $g \partial d / \partial x$ continues to dominate the shallow water equations despite the induced time dependence. If we partition the flow over the obstacle between basic $(U, H)$ and wave induced $\left(u^{\prime}, \eta\right)$ fields, the $x$-momentum equation reads

$$
\frac{\partial u}{\partial t}+U \frac{\partial u^{\prime}}{\partial x}+u^{\prime} \frac{\partial U}{\partial x}=-g \frac{\partial \eta}{\partial x} .
$$

If the first term is small with respect to any of the remaining three, the quasisteady assumption is valid. The ratio of the first to the second is $L / U T$, where $L$ is the obstacle half length. For an incident wave of length $\lambda$, the period $T$ is $\lambda /\left[u+(g H)^{\frac{1}{2}}\right]$, and thus $L / U T=(L / \lambda)\left(1+F_{d}\right)=0(L / \lambda)$. A good rule of thumb, then, is that the incident wave length be $\gg L$ for quasisteadiness. This rule breaks down if $F_{d} \rightarrow 1$, for then the wave length $\lambda_{R}$ of the reflected wave becomes smaller than $L$ and the above scaling arguments must be reconsidered. As mentioned earlier, such situations are unlikely to occur in the deep ocean. Our calculations also assume that bores or other nonlinear disturbances which might be generated downstream of the sill cannot reach the sill. The conditions under which this assumption holds involve analysis of possible hydraulic jumps in the lee of the sill and are more difficult to formulate in simple terms. For further discussion of the above assumptions in connection with a two-layer flow the reader is referred to Helfrich (1995) or Baines (1995).

\section{Deep sill with rotation}

We now consider the case of rotating channel flow with uniform potential vorticity, $q$. 'Uniform' means that $q$ does not vary with $x$ or $y$ over the region extending from the 
artificial boundary $(x=0)$ to the sill, whereas slow variations in $t$ are permitted. Thus the numerically calculated value of $q$ just upstream of $x=0$ at time $t$ is assumed to be the value from that position to the sill. Although $y$-variations in this numerically calculated $q$ would lead to a dilemma, the resolution afforded by most current GCM's makes it difficult to distinguish uniform from nonuniform potential vorticity across deep passages as narrow as the Denmark Strait, Faroe Bank Channel or Vema Channel.

The assumption of spatially uniform $q$ with slow temporal variations specifically means that a wave propagating from the artificial boundary to the sill and being reflected back to the boundary sees a fixed value of $q$. If $c_{i}$ and $c_{r}$ are the average speeds of the incident and reflected wave and $L$ is the boundary-to-sill distance, then the travel time of the signal over the path just described is approximately $L / c_{r}+L / c_{i}=$ $O\left(L / c_{r}\right)$ (since $\left|c_{r}\right|$ is normally $\left\langle\left|c_{i}\right|\right)$. The time period of change of $q$ within the region is the advection time $L / U$, where $U$ is a typical $x$-velocity. Our approximation thus requires that $U / c_{r} \ll 1$, or roughly that the average Froude number of the approaching flow be small, a situation typically satisfied.

We continue to assume a rectangular cross-section, although width and bottom elevation changes in the approach region are now formally included. Although Riemann invariants can, in principle, be found for channels with continuous $y$-variations in bottom elevation the technical details are more difficult (e.g. Borenäs and Lundberg, 1986). Though somewhat artificial, the rectangular-cross-section model does provide the basic ingredients for critical control of an abyssal flow. For numerical purposes the cross-section need only be rectangular only one grid point upstream of $x=0$.

The development of the unsteady rotating-channel equations for uniform potential vorticity is laid out in Pratt (1983) and is based on the formalism introduced by Gill (1977) for steady hydraulics problem. Due to the increased number of dimensional parameters it is easiest to present the results in dimensionless form using $\lambda$, the typical wave length, as a scale for $x$-variations, $D$ as a depth scale, $\sqrt{g D} / f$ as a width scale ( $f$ being the constant Coriolis pardmeter), $\lambda /(g D)^{\frac{1}{2}}$ as a time scale, $(g D) / f \lambda$ as scale for $v$ (the $y$-velocity) and $(g D)^{\frac{1}{2}}$ for $u$. Under the assumption of gradual variations along the channel $\left(\lambda \gg(g D)^{\frac{1}{2}} / f\right)$ the potential vorticity is approximated by

$$
q=\frac{1-\partial u / \partial y}{h}
$$

all quantities now being dimensionless. Furthermore the along-stream velocity is approximately geostrophic:

$$
u=-\frac{\partial h}{\partial y}
$$

a fact which allows Eq. (18) to be rewritten as

$$
\frac{\partial^{2} h}{\partial y^{2}}-q h=-1 \text {. }
$$


The solution to Eq. (20) and the corresponding geostrophic velocity can be written in the form

$$
h=q^{-1}+\delta h(x, t) \frac{\sinh \left(q^{\frac{1}{2}} y\right)}{\sinh \left[q^{\frac{1}{2}} W(x) / 2\right]}+\left[\bar{h}(x, t)-q^{-1}\right] \frac{\cosh \left(q^{\frac{1}{2}} y\right)}{\cosh \left[q^{\frac{1}{2}} W(x) / 2\right]}
$$

and

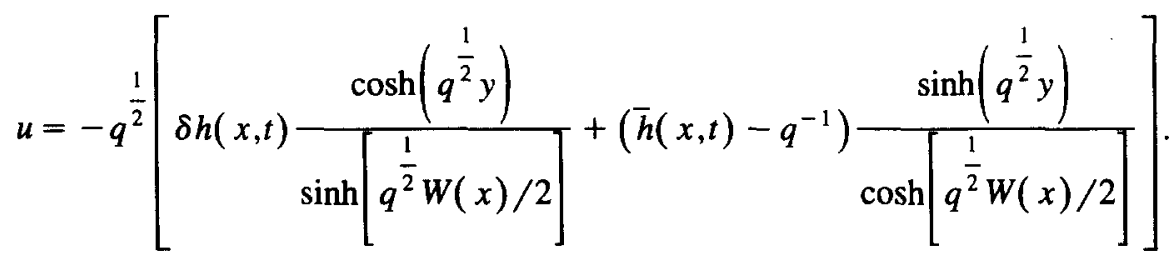

The integration 'constants' $\delta h$ and $\bar{h}$ have been chosen to represent half the difference and sum of the depths along the side walls lying at $y= \pm \frac{1}{2} W(x)$, that is

$$
\begin{aligned}
& \bar{h}=\frac{h[x, W(x) / 2, t]+h[x,-W(x) / 2, t]}{2} \\
& \delta h=\frac{h[x, W(x) / 2, t]-h[x,-W(x) / 2, t]}{2} .
\end{aligned}
$$

The $x$ - and $t$-dependence of the solutions are obtained by evaluating the $x$-momentum equations on both side walls, applying the boundary conditions $v\left[x, \pm \frac{1}{2} W(x), t\right]=$ $\pm u[x, \pm W(x), t] d(W / 2) / d x$ and substituting the expressions Eq. (21) and Eq. (22) for $u$ and $h$. Taking the difference and sum of the two results leads to

$$
\begin{aligned}
& \frac{\partial}{\partial t}(\delta h)-q^{\frac{1}{2}} T^{-1} \delta h \frac{\partial}{\partial x}(\delta h)+\left[q^{\frac{1}{2}} T^{3}\left(q^{-1}-\bar{h}\right)-T q^{-\frac{1}{2}}\right] \frac{\partial \bar{h}}{\partial x} \\
& \quad=T q^{-\frac{1}{2}}\left[\frac{d d(x)}{d x}-\frac{1}{4} q\left(u_{+} h_{+}-u_{-} h_{-}\right) \frac{d W}{d x}\right] \\
& \frac{\partial \bar{h}}{\partial t}-q^{\frac{1}{2}} T^{-1} \bar{h} \frac{\partial}{\partial x}(\delta h)-q^{\frac{1}{2}} T^{-1} \delta h \frac{\partial \bar{h}}{\partial x}=-\frac{1}{4} q^{\frac{1}{2}} T^{-1}\left(u_{+} h_{+}+u_{-} h_{-}\right)
\end{aligned}
$$

where

$$
T=\tanh \left[q^{\frac{1}{2}} W(X) / 2\right],
$$

$d(x)$ denotes the bottom elevation, and the subscripts ' + ' and ' - ' denote quantities evaluated at $y= \pm W(x) / 2$. 
Using the method described by Whitham (1974), Eq. (23) and Eq. (24) may be cast in the form

$$
\begin{aligned}
\frac{d \pm R \pm}{d t}= & -\frac{d[d(x)]}{d x}+[q(\bar{u} \delta h+\bar{h} \delta u) \\
& \left.-q^{\frac{1}{2}} T^{-1}\left(q^{\frac{1}{2}} T^{-1} \delta h-c_{ \pm}\right) \bar{h}^{-1}(\bar{u} \bar{h}+\delta u \delta h)\right] \frac{d W}{d x}
\end{aligned}
$$

where

$$
\begin{aligned}
& \frac{d \pm}{d t}=\frac{\partial}{\partial t}+c_{ \pm} \frac{\partial}{\partial x} \\
& c_{ \pm}=-q^{\frac{1}{2}} T^{-1} \delta h \pm \bar{h}^{\frac{1}{2}}\left[1-T^{2}(1-q \bar{h})\right]^{\frac{1}{2}} \\
& R_{ \pm}=-q^{\frac{1}{2}} T^{-1} \delta h \pm \int^{\bar{h}} r(\alpha) d \alpha \\
& r(\bar{h})=\bar{h}^{-\frac{1}{2}}\left[1-T^{2}(1-q \bar{h})\right]^{\frac{1}{2}} \\
& \bar{u}=-q^{\frac{1}{2}} T^{-1} \delta h
\end{aligned}
$$

and

$$
\delta u=q^{\frac{1}{2}} T\left(q^{-1}-\bar{h}\right) .
$$

As with the nonrotating case, $R_{ \pm}$are conserved along characteristic curves $d x / d t=c_{ \pm}$ provided $d(x) / d x=d W / d x=0$. If the approach section of the channel (upstream of $x=0$ ) converges, then the rate of change of $R_{ \pm}$obeys Eq. (25).

There are several points to be made about these relations. First, the term $-q^{\frac{1}{2}} T^{-1} \delta h$ appearing in the signal speeds $c_{ \pm}$is nothing more than the average $\bar{u}$ of the $x$-velocities at the two side walls. Second, the signals are essentially nonlinear Kelvin waves modified by the cross-channel shear and depth variation. In the limit of slow rotation, $T \rightarrow 0$, Eq. (26) reduces to

$$
c_{ \pm}=\bar{u} \pm \bar{h}^{\frac{1}{2}}
$$

the dimensionless form of the nonrotating result. Third, the governing equations are valid only as long as the fluid depth remains finite across the entire channel width. It is well known that rotating channel flow can separate from the wall at $y=W / 2$ forming a free edge. As argued by Stern (1980) and supported in the laboratory experiments of Pratt (1987) the presence of a free edge in rectangular geometry implies supercritical flow, which is inconsistent with the upstream state of a hydraulically controlled flow. This result is tied into the fact that Kelvin waves no longer exist along the wall at 
$y=W / 2$ once separation occurs. We will therefore consider only nonseparated flow in the upstream sections. Finally, the indefinite integral in the definition (Eq. (27) and Eq. (28)) of the Riemann invariant $R_{ \pm}$can be evaluated in closed form, the result being

$$
\begin{aligned}
\int^{\bar{h}} r(\alpha) d \alpha= & \bar{h}^{\frac{1}{2}}\left[1-T^{2}(1-q \bar{h})\right]^{\frac{1}{2}} \\
& +\frac{1}{2} T^{-1} q^{-\frac{1}{2}}\left(1-T^{2}\right) \ln \left\{2 T q^{\frac{1}{2}} \bar{h}^{\frac{1}{2}}\left[1-T^{2}(1-q \bar{h})\right]^{\frac{1}{2}}\right. \\
& \left.+2 T^{2} q \bar{h}+1-T^{2}\right\} .
\end{aligned}
$$

The dimensional forms of the Kelvin wave speeds and Riemann invariants are

$$
\begin{aligned}
c_{ \pm}^{*}=\sqrt{g D} \lambda=\bar{u}^{*} \pm\left(g \bar{h}^{*}\right)^{\frac{1}{2}}\left[1-T^{2}\left(1-q^{*} \bar{h}^{*} / f\right)\right]^{\frac{1}{2}} \\
R_{ \pm}^{*}=\sqrt{g D} R=\bar{u}^{*} \pm\left(g \bar{h}^{*}\right)^{\frac{1}{2}}\left[\left[1-T^{2}\left(1-q^{*} \bar{h}^{*} / f\right)\right]^{\frac{1}{2}}\right. \\
+\frac{1}{2} T^{-1}\left(f / q^{*} \bar{h}^{*}\right)^{\frac{1}{2}} \ln \left\{2 T\left(\frac{q^{*} \bar{h}^{*}}{f}\right)^{\frac{1}{2}}\left[1-T^{2}\left(1-q^{*} \bar{h}^{*} / f\right)\right]^{\frac{1}{2}}\right. \\
\left.\left.+\frac{2 T^{2} q^{*} \bar{h}^{*}}{f}+1-T^{2}\right\}\right]
\end{aligned}
$$

At the artificial boundary $x=0$ the conditions to be satisfied stem from conservation of mass and energy between $x=0$ and the sill as well as the condition $c_{-}=0$ of criticality at the sill. By the geostrophic relation the quantity

$$
Q=-\delta h \bar{h}
$$

which is one-half the total volume flux is conserved, as is the quantity

$$
\vec{B}=\frac{1}{2} q T^{-2}(\delta h)^{2}+\frac{1}{2} q T^{2}\left(q^{-1}-\bar{h}\right)^{2}+\bar{h},
$$

the average of the Bernoulli functions evaluated at each wall. As shown by Pratt (1983) (specifically his (4.2) and (4.3) as adapted from Gill, 1977), combining these relationships with the statement of sill criticality lead to the following constraints

$$
\bar{h}_{s}^{4}+q^{-1}\left(T_{s}^{-2}-1\right) \bar{h}_{s}^{3}-T_{s}^{-4} Q^{2}=0
$$

and

$$
\bar{B}-\frac{1}{2} q T_{s}^{2}\left(q^{-1}-\bar{h}_{s}\right)\left(q^{-1}-2 \bar{h}_{s}\right)-\frac{3}{2} \bar{h}_{s}=d_{s} .
$$


One can now set the machinery of Section 2 in motion. In principle the average depth $\bar{h}_{s}$, over the sill could be eliminated from Eq. (34) and Eq. (35), forming a single equation (analogous to Eq. (6)) for $Q$ and $\bar{B}$. By evaluating $Q$ and $\bar{B}$ at the artificial boundary one would then have a single equation relating $\delta h(0, t)$ to $\bar{h}(0, t)$. In principle the desired relationship between $R_{+}$and $R$ could be found by writing $\delta h$ and $\bar{h}$ in terms of $R_{+}$and $R_{-}$using Eq. (27) and Eq. (28) and substituting into the former result. The algebra involvē would be nightmarish and one would be better off writing a numerical algorithm to accomplish the task. An attractive alternative would be to use the results of the linear reflection coefficient calculation presented next.

If a boundary condition on $v$ is required, the approximation $v=0$ might suffice since the artificial boundary is within the deep strait where the flow is nearly unidirectional.

\section{Kelvin wave reflection}

The calculation of the reflection coefficient for linear Kelvin waves parallels the steps laid out in Section 3 for the nonrotating case and, since the algebra is much more tedious, we only outline the steps. To start with, denote the background values of $\bar{h}$ and $\delta h$ by $\bar{H}$ and $\delta H$, and let $\bar{U}=-q^{\frac{1}{2}} T^{-1} \delta H$. It is now assumed that $d W / d x=d d / d x=0$ at $x=0$, though the channel may widen or shoal elsewhere. Next, using the definitions Eq. (27) and Eq. (28) for the rotational Riemann invariants, define $\bar{u}_{I, R}$ and $\eta_{I, R}$ by

$$
R_{+}=2 \bar{u}_{l}+\bar{U}+\int^{\bar{H}} r(\alpha) d \alpha=2 \int^{\bar{H}+\bar{\eta}_{l}} r(\alpha) d \alpha+\bar{U}-\int^{\bar{H}} r(\alpha) d \alpha
$$

and

$$
R_{-}=2 \bar{u}_{R}+\bar{U}-\int^{\bar{H}} r(\alpha) d \alpha=-2 \int^{\bar{H}+\bar{\eta}_{R}} r(\alpha) d \alpha+\bar{U}+\int^{\bar{H}} r(\alpha) d \alpha
$$

where again $\bar{U}+\bar{u}_{I, R}$ and $\bar{H}+\bar{\eta}_{I, R}$ can be shown to be the average of the side wall velocity and depth for incident or reflected waves moving into regions of undisturbed (background) flow. For $\bar{\eta}_{I, R} \ll \bar{H}$ we have the linear approximations

$$
\begin{aligned}
\bar{u}_{l} & =\int^{\bar{H}+\bar{\eta}_{l}} r(\alpha) d \alpha-\int^{\bar{H}} r(\alpha) d \alpha=\int_{\bar{H}}^{\bar{H}+\bar{\eta}_{I}} r(\alpha) d \alpha \\
& =\int_{\bar{H}}^{\bar{H}+\bar{\eta}_{I}}\left[r(\bar{H})+\alpha r^{\prime}(\bar{H})+\ldots\right] d \alpha \\
& \cong r(\bar{H}) \bar{\eta}_{I} \\
& =\bar{H}^{-\frac{1}{2}}\left[1-T^{2}(1-q \bar{H})\right]^{\frac{1}{2}} \bar{\eta}_{I}
\end{aligned}
$$

and similarly

$$
\bar{u}_{R} \cong-r(\bar{H}) \bar{\eta}_{R}=-\bar{H}^{-\frac{1}{2}}\left[1-T^{2}(1-q \bar{H})\right]^{\frac{1}{2}} \bar{\eta}_{R} .
$$

To calculate the reflection coefficient, one first uses Eq. (32) and Eq. (33) to substitute for $\bar{B}$ and $Q$ in Eq. (34) and Eq. (35). Next, substitute $\bar{U}+\bar{u}_{I}+\bar{u}_{R}$ for 
$-q^{\frac{1}{2}} \mathrm{~T}^{-1} \delta h, \bar{H}+\bar{\eta}_{I}+\bar{\eta}_{R}$ for $\bar{h}$, and $\bar{H}_{s}+\bar{h}_{s}^{\prime}$ for $h_{s}$, in the new version of Eq. (34) and Eq. (35) and linearize. Finally, eliminate $\bar{h}_{s}^{\prime}$, between the two resulting expressions and rearrange, obtaining

$$
R_{c}=\frac{\bar{\eta}_{R}}{\bar{\eta}_{I}}=\frac{(1-G)(1+F)}{(1+G)(1-F)}
$$

where $F$ is the Froude number of the approach flow:

$$
F=\frac{\bar{U}}{\bar{H}^{\frac{1}{2}}\left[1-T^{2}(1-q \bar{H})\right]^{\frac{1}{2}}},
$$

$G$ is defined by

$$
G=\left(\frac{\bar{H}_{s} T_{s}}{\bar{H} T}\right)^{2} F^{-1}
$$

and the subscript $s$ denotes values evaluated at the sill. One can recover the nonrotating result by reducing either $W$ or $q$ to zero, corresponding to $T \rightarrow 0$, in which case $G \rightarrow F^{\frac{1}{3}}\left(W_{s} / W\right)^{\frac{2}{3}}$ and Eq. (13) is obtained. Also, one can obtain a reflection coefficient for displaced volume in the same manner as done in the nonrotating case. The result is

$$
R_{v}=\left(\frac{1-F}{1+F}\right) R_{c}
$$

where $R_{v}$ is the ratio of displaced volume of the reflected and incident waves.

The simplicity of the formula for $R_{c}$ is deceptive. In addition to the potential vorticity $q$, the geometric parameters $T$ and $T_{s}$, and the dynamical quantity $\bar{H}$ at the artificial boundary, the formula depends on $\vec{H}_{s}$, the critical value of $\bar{H}$ at the sill. In order to express $R_{c}$ entirely in terms of geometrical and upstream dynamical information $\bar{H}_{s}$ must be related to the upstream base flow. To do so one must solve the quartic equation obtained by replacing $\bar{h}_{s}$ by $\bar{H}_{s}$ and $Q$ by $\bar{H} \delta H$ in Eq. (34), namely $\bar{H}_{s}^{4}+q^{-1}\left(T_{s}^{-2}-\right.$ 1) $\bar{H}_{s}^{3}-T_{s}^{-4}(\bar{H} \delta H)^{2}=0$. The end result is that $R_{c}$ depends on the five quantities $q, T$, $T_{s}, \delta H$ and $\bar{H}$. Alternatively, the sill elevation $d_{s}$ may be substituted for any of the five through the use of Eq. (33) and Eq. (35).

To display the results (see Figs. 7-10) the values of $R_{c}$ are plotted in $(\bar{H}, \delta H)$ planes corresponding to particular values of $q, T$, and $T_{s}$. In addition to the contours of constant $R_{c}$ (thin solid lines) the constant $d_{s}$ contours (dashed lines) are also shown in each plane. Thus the potential vorticity and geometry are completely specified by moving along a dashed line in any of the figures. One must take caution with this approach as not all portions of the $\bar{H},-\delta H$ plane correspond to acceptable background flows. If the flow is separated at the sill section, which occurs when

$$
-\delta H \bar{H}<\frac{T_{s}^{4}}{q^{2}\left(1+T_{s}^{2}\right)^{2}}
$$


(Gill, 1977) or at the artificial boundary, corresponding to

$$
-\delta H>\bar{H},
$$

one may wish to disregard the solution as being inconsistent with critical control as argued earlier. Although the final word on the acceptability of separated flows in rectangular geometry has yet to be written, we will at least identify the regions in which the flows occur. In addition, the approach flow must be subcritical $F<0$, which leads to

$$
-\delta H<q^{-\frac{1}{2}} T \bar{H}^{\frac{1}{2}}\left(1-T^{2}+T^{2} q \bar{H}\right)^{\frac{1}{2}}
$$

in view of Eq. (39) and the relation $\bar{U}=-q^{\frac{1}{2}} T^{-1} \delta H$. The three boundaries implied by Eq. (40), Eq. (41) and Eq. (42) are plotted in Fig. 6 for $q=1, T=0.5$ and $T_{s}=0.25$, the shaded region corresponding to acceptable solutions.

Figs. 7-9 show $R_{c}$ for geometries with sills but no width contraction $\left(T=T_{s}\right)$ and with $q=1$. For Fig. $7, T$ and $T_{s}$ have been assigned the value 0.8 , corresponding to a channel width of 2.2 deformation radii. (The nondimensional deformation radius is $q^{-\frac{1}{2}}$, as suggested by the arguments of the hyperbolic functions in the formula Eq. (21) for the cross-channel structure.) The effect of rotation is thus quite strong in this case as the incident Kelvin wave, trapped on the 'right' wall $(y=-W / 2)$, barely 'feels' the reflected Kelvin wave, trapped on the opposite wall. As shown in Fig. 7, $0<R_{c}<1$ over most of the subspace of acceptable solutions although some cases of $R_{c}>1$ occur near the boundary corresponding to critical flow. This is a departure from the nonrotating case, where $R_{c}>1$ occurred only for geometries with both width and elevation variations. In the present case $R_{c} \rightarrow \infty$ as the critical curve is approached.

If the values of $T$ and $T_{s}$ are simultaneously reduced, ostensibly reducing the effects of rotation, a remarkable change in the behavior of $R_{c}$ occurs. A threshold value

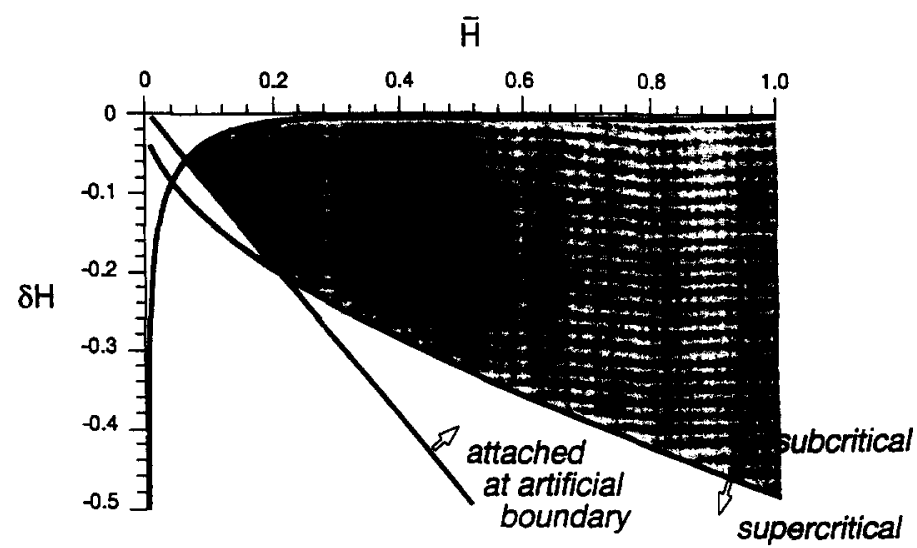

Fig. 6. The shaded region in the space $(\bar{H}, \delta H)$ of the upstream flow space indicates solutions which are critically controlled at the sill, subcritical upstream, and attached to the side walls at both the artificial boundary and the sill. The three bounding curves correspond to Eqs. (34)-(36) with $q=1, T=0.5$ and $T_{s}=0.25$. 


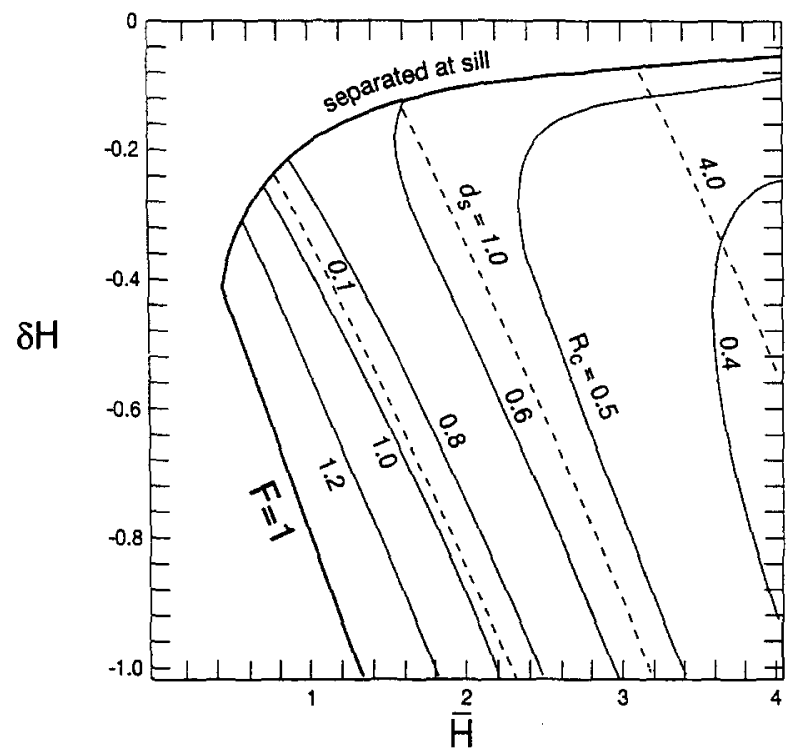

Fig. 7. Reflection coefficient for rotating channel flow with uniform potential vorticity $q=1$ and $T=T_{s}=0.8$. The dashed lines are contours of dimensionless sill elevation $d_{s}$. The bounding curves (bold) represent critical flow at the artificial boundary $(F=1)$ or separated flow at the sill.

$T=T_{s}=0.5$ is first encountered at which $R_{c}$. becomes finite at all points of the solution subspace (Fig. 8). Moreover, no cases of $R_{c}>1$ exist and $0<R_{c}<0.3$ over most of the plane. If $T$ and $T_{s}$ are increased slightly above the value 0.5 , which corresponds to a

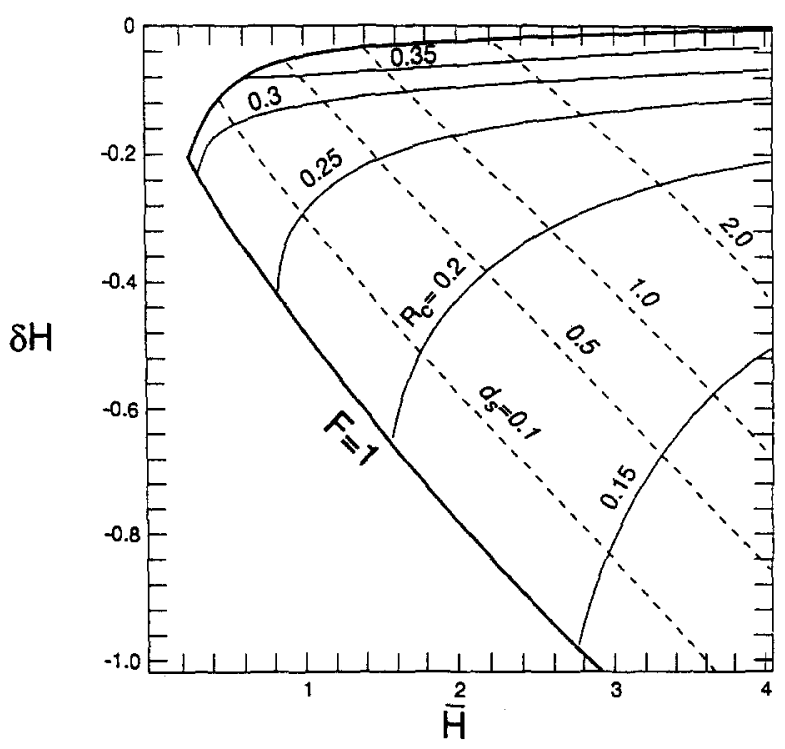

Fig. 8. Same as Fig. 7 but with $T=T_{s}=0.5$. 


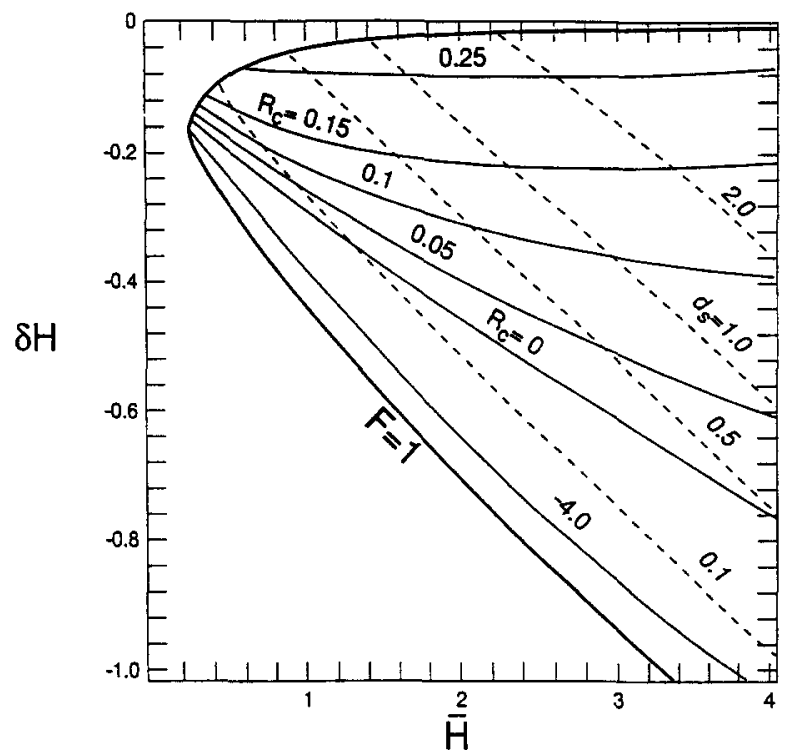

Fig. 9. The same as Fig. 7 but with $T=T_{s}=0.46$.

channel width of 1.1 deformation radius, the singularities in $R_{c}$ reappear and the solution takes on the general appearance of Fig. 7 .

For $T$ and $T_{s}$ below the threshold value 0.5 , both positive and negative values of $R_{c}$ appear, with $R_{c} \rightarrow-\infty$ near the boundary of critical flow. Fig. 9 shows an example with $T=T_{s}=0.46$ corresponding to a channel width of 1.0 deformation radius. A contour

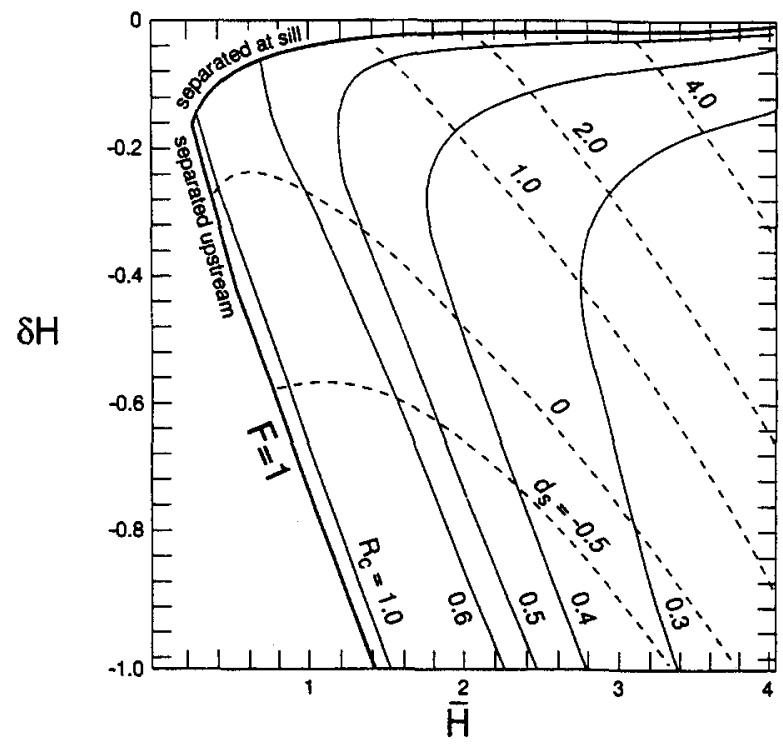

Fig. 10. The same as Fig. 7 but with $T=0.8$ and $T_{s}=0.46$. 
$R_{c}=0$ separates negative values below from positive and finite values above. Comparing this plot with Fig. 7 leads to the conclusion that $\left|R_{c}\right|$ is smaller for weaker rotation, at least over most of the solution space plotted. This result runs counter to the intuition that wider channels (with incident and reflected Kelvin waves isolated from each other) should produce small values of $\left|R_{c}\right|$. However, wider rotating channels also require higher sills for critical control and this contributes to higher values of $\left|R_{c}\right|$.

When width contractions are allowed in addition to sills, a wide range of behavior is possible. Here we simply describe a case which is typical of ones likely to arise in geophysical situations. The channel narrows from a width $W=2.2$ deformation radii (or $T=0.8$ ) at the artificial boundary to $W_{s}=1$ deformation radius at the sill. As shown in Fig. 10 the $R_{c}$ values lie in the range $0<R_{c}<0.25$ over most of the domain with $R_{c}>1$ only in a narrow strip near the boundary for critical flow. This strip also lies within a region corresponding to negative sill elevations, $d_{s}<0$. In summary, GCM simulations for a deep channel with this width structure and with positive sill elevations would encounter small, positive $R_{c}$ values.

\section{Discussion}

Since the primary thrust of this work is establishing guidelines for imbedding deep hydraulic controls in numerical models, we summarize the procedure for doing so using the rotating channel model with uniform potential vorticity. One must first identify a layer or level of the numerical model which corresponds to overflowing fluid and one must be prepared to treat it locally as a reduced gravity layer (with $1 \frac{1}{2}$-layer dynamics). One must further arrange the local bottom topography so that the approach to the sill has the configuration of a deep channel with gradually narrowing rectangular cross sections. This topography terminates at an artificial boundary (at $x=0$ ) a short distance upstream of the actual sill. The investigator must choose the sill width $\left(W_{s}\right)$ and elevation $\left(d_{s},\right)$ and the width $W$ at the artificial boundary as deemed consistent with the actual topography.

The artificial boundary exists only in the lowest layer (or level) of the model and only this layer is removed from explicit numerical resolution and treatment over the rapidly varying sill topography. The numerical model generally spans both the upstream and downstream basin and the lowest layer must somehow be joined back in with the downstream numerics. We suggest doing so with an outflow model of the type introduced by Price and Baringer (1994) or Jungclaus and Backhaus (1994). Numerical treatment of the overlying fluid between the two basins can be carried out using something like the normal algorithm, provided that this fluid is decoupled from the overflow. Further remarks on the situation where the layers are not decoupled are made below.

Given the entire model state at time $t_{0}$, incorporation of the hydraulic control amounts to the prediction of the state at the artificial boundary at time $t_{0}+\Delta t$. To do so, one calculates the Riemann invariant $R_{+}$(Eq. (27)) at one or two grid points upstream of $x=0$ by estimating the potential vorticity $q$, and the one half the sum and difference $\bar{h}$ and $\delta h$ of the side wall layer thicknesses. These values of $R_{+}$are then projected 
forward in time along characteristic curves $d x / d t=c_{+}$(Eq. (26)) to the artificial boundary and the value of $R_{+}\left(0, t_{0}+\Delta t\right)$ is interpolated from the results. (If the channel width is constant the characteristic $R_{+}$remains constant as well, otherwise Eq. (25) is used to calculate its rate of change.) The potential vorticity $q$ is assumed constant along the characteristic.

Once $R_{+}\left(0, t_{0}+\Delta t\right)$ is known one can solve for $R\left(0, t_{0}+\Delta t\right)$ and hence $\delta h\left(0, t_{0}+\right.$ $\Delta t)$ and $\bar{h}\left(0, t_{0}+\Delta t\right)$ as described at the end of Section 4 . This procedure would give the fully nonlinear result. An alternative procedure requiring considerably less complexity would be to define a background state $\bar{H}$ and $\bar{U}\left(=-q^{\frac{1}{2}} T^{-1} \delta H\right)$ at the artificial boundary. From $\bar{H}, \bar{U}$, and $R_{+}(0, t+\Delta t)$ one calculates perturbation average velocity $\bar{u}_{l}$ associated with incident signals using Eq. (36a). A corresponding $\bar{\eta}_{l}$ is computed from Eq. (36b). At this point the definition of the background should be checked to be sure that $\left|\bar{u}_{l}\right| \ll|\bar{U}|$ and $\left|\bar{\eta}_{I}\right| \ll|\bar{H}|$ so that linear wave reflection theory can be applied. Finally, the reflected signal $\bar{\eta}_{R}$ and $\bar{u}_{R}$ is computed from Eq. (37b) and Eq. (38) and the resulting total boundary values are

$$
\begin{aligned}
& \bar{h}\left(0, t_{0}+\Delta t\right)=\bar{H}+\bar{\eta}_{r}+\bar{\eta}_{I} \\
& \delta \bar{h}\left(0, t_{0}+\Delta t\right)=-q^{-\frac{1}{2}} T\left(\bar{U}+\bar{u}_{R}+\bar{u}_{I}\right) .
\end{aligned}
$$

The background flow may be varied from one time step to the next.

We have identified a number of situations in which the reflection coefficient may become invalid. The first of these is the occurrence of hydraulically critical flow at $x=0$. For realistic forcing and bathymetry, such an event would be a severe departure from reasonable views of the abyssal circulation that one would have to call the numerical model physics into question. The second situation corresponds to separated flow at $x=0$. For the parameter range explored in Section 5 , this condition implies that the approach flow is either supercritical or only slightly subcritical (e.g. compare the corresponding curves in Fig. 6 for example), and the same comments apply. Finally it is possible that separated flow at the sill might be encountered, which is much more likely than the first two possibilities. Here we simply note that the $(\bar{H}, \delta \bar{H})$ space occupied by such a regime in any of Figs. 7-10 is small and that the variation of $R_{c}$ over that area is weak. Thus, one might simply attribute separated sill flow to a slight error in the model physics and continue to use the value of $R_{c}$ as determined by Eq. (38) knowing that the result is insensitive to the state of separation.

The above remarks propose imperfect solutions to the difficulties presented by separated flow. In principle, a better approach would be to develop a theory for time-dependent rotating channel flow with continuously varying topography, so that the distinction between separated and nonseparated flow would be lost. Such a model could build on the work of Borenäs and Lundberg (1986) in an attempt to find Riemann invariants.

Often, the local representation of the overflow as a single, reduced-gravity layer will be inadequate. For example, Hogg (1983) has attempted to model the Vema Channel using $2 \frac{1}{2}$-layer stratification. In such cases, the governing principles described herein may or may not be applicable. If the controlling wave mode can propagate in both 
directions (i.e. the flow is subcritical with respect to that mode) at all points on one side (say $x<0$ ) of the sill, then our methods can be applied in $x<0$. An example would be the 'submaximal', two layer exchange flow solutions identified by Farmer and Armi (1986) in which the lower layer spills over a sill from left to right and forms an internal hydraulic jump (also see Dalziel, 1990, 1991 and Pratt and Armi, 1990). The upper layer flows right-to-left but forms no jump after it passes the sill. Waves approaching the sill from the left are partially reflected in a manner analogous to our simpler model and left is therefore the 'upstream' direction. This flow could be treated using our methods, although the artificial boundary would span both layers. Conversely, the two-layer flow might contain 'virtual' or 'approach' controls or hydraulic jumps to the left of the obstacle, with associated subcritical/supercritical transitions. In this case incident waves would never reach the sill and our method would fail.

Finally, we point out that an alternative procedure for approximating the effects of rotating hydraulic controls in numerical models might be to apply transport bounds recently developed by Killworth (1994). Here the physics of critical control would be present only to the extent that the deep transport would be prevented from exceeding certain limits. The procedure for implementation has not been established. One advantage of this approach is that the bounds are valid for much more general topography and potential vorticity than considered herein.

\section{Acknowledgements}

This work was supported by NSF grant OCE-9115359. The authors thank Deborah Taylor for preparing this manuscript.

\section{References}

Baines, P.G., 1995. Topographic Effects in Stratified Flows Cambridge University Press, Cambridge, 482 pp. Borenäs, K.M. and Lundberg, P.A., 1986. Rotating hydraulics of flow in a parabolic channel. J. Fluid Mech., 167: 309-326.

Dalziel, S.B., 1990. Rotating two-layer sill flows. In: L.J. Pratt (Ed.), The Physical Oceanography of Sea Straits. NATO-ASI Set., Kluwer, Dordrecht, pp. 343-371.

Dalziel, S.B., 1991. Two-layer hydraulics-a functional approach. J. Fluid Mech., 223: 135-163.

Farmer, D.M. and Armi, L., 1986. Maximal two-layer exchange over a sill and through a combination of a sill and contraction with barotropic net flow. J. Fluid Mech., 164: 53-76.

Gill, A.E., 1977. The hydraulics of rotating-channel flow. J. Fluid Mech., 80: 641-671.

Grimshaw, R. and Smyth, N., 1986. Resonant flow of a stratified fluid over topography. J. Fluid Mech., 169: $429-464$.

Hayashi, Y.-Y. and Young, W.R., 1987. Stable and unstable shear modes of rotating parallel flows in shallow water. J. Fluid Mech., 184: 477-504.

Helfrich, K.R., 1995. Time dependent two-layer hydraulic exchange flow. J. Phys. Oceanogr., 25: 359-373.

Hogg, N., 1983. Hydraulic control and flow separation in a multilayered fluid with applications to the Vema Channel. J. Phys. Oceanogr., 13: 695-708.

Jungclaus, J.H. and Backhaus, J.O., 1994. Application of a transient reduced gravity plume model to the Denmark Strait Overflow. J. Geophys. Res., 99(C6): 12375-12396.

Killworth, P.D., 1994. On reduced-gravity flow through sills. Geophys. Astrophys. Fluid Dyn., 75: 91-106. 
Pratt, L.J., 1983. On inertial flow over topography. Part 1. Semigeostrophic adjustment to an obstacle. J. Fluid Mech., 131: 195-218.

Pratt, L.J., 1984. A time-dependent aspect of hydraulic control in straits. J. Phys. Oceanogr., 14: 1414-1418.

Pratt. L.J., 1987. Rotating shocks in a separated laboratory channel flow. J. Phys. Oceanogr., 17: 483-491.

Pratt, L.J. and Armi, L., 1990. Two-layer rotating hydraulics: strangulation, remote and virtual controls. Pure Appl. Geophys., 133 (4): 587-617.

Price, J.F. and Baringer, M.O., 1994. Outflow and deep water production by marginal seas. Prog. Oceanogr., 33: $161-200$.

Ripa, P., 1989. On the stability of ocean vorticies. In: J.J.C. Nihoul and B.M. Jamart (Eds.), Mesoscale/Synoptic Coherent Structures in Geophysical Turbulence. Elsevier Oceanographic Series, Elsevier Science, Amsterdam, pp. 167-179.

Stern, M.E., 1980. Geostrophic fronts, bores, breaking and blocking waves. J. Fluid Mech., 99: 687-703.

Whitham, G.B., 1974. Linear and Nonlinear Waves. Wiley \& Sons, New York, 636 pp. 